\title{
Quantum Mechanics of a Simulated Trihydrogen Dication
}

\author{
ML Glasser \\ Departamento de Física Teórica, Atómica y Óptica, \\ Universidad de Valladolid 47071 Valladolid, Spain \\ Department of Physics, Clarkson University, \\ Potsdam, NY 13699-5820 (USA)
}

\begin{abstract}
The Schroedinger equation is solved exactly within the Born-Oppenheimer

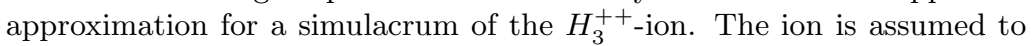
form an isosceles triangle and the ground state energy is obtained over its geometrical parameter space. No multi-center molecular integrations are required. We indicate how the approximation to the actual molecule can be improved systematically.
\end{abstract}

pacs 31.15.Ar; 02.70.Wz; 31.50.Bc

\section{Introduction}

The Trihydrogen cation $H_{3}^{+}$was identified, by mass spectroscopy, in 1911 by J.J. Thomson[1]. Twelve years later Hogness and Lunn[2] found that it could be produced by the proton exchange $\mathrm{H}_{2}^{+}+\mathrm{H}_{2} \rightarrow \mathrm{H}_{3}^{+}+\mathrm{H}$ and would readily lose an electron. Subsequently it was found that $H_{3}^{+}$is present in interstellar clouds and is among the most abundant molecular species in the universe. This led to the question of the stability of the dication $\mathrm{H}_{2}^{++}$, which has remained somewhat controversial to the present, though the consensus is that it is unstable.

The first quantum treatment was by Gordadse[3, 4] in (1935), who assumed the protons were fixed, equally spaced along a straight line or formed an equilateral triangle. He used the variational method, as have all subsequent studies, based on a one-parameter trial function built from the hydrogen 1s-state. In spite of the simplicity of the trial functions, he found several of the multi-center integrations intractable, requiring not too well controlled approximations, and concluded that neither configuration was stable. About the same time C.A. Coulson carried out a LCMO study of $H_{3}^{++}$[5]. He used only a single molecular orbital and his energy values lie somewhat higher than those in [4]. The difficulty of these multi-center integrations have continued to dog such calculations and may be what prompted Eyring's comment that $H_{3}^{+}$is "the scandal of modern chemistry" [6]. The first extensive study of $\mathrm{H}_{3}^{++}$using electronic digital computers was by H. Conroy in 1964 [7,8,9,10], who devised an insightful set of variational wave functions and used Monte-Carlo algorithms for the integrations, remarking that "these integrations presented grave obstacles". This and subsequent calculations $[11,12,13,14,15]$, mostly confined to the linear and 
equilateral triangle configurations, have upheld Gordadse's conclusion that the ion is unstable. An attempt to produce the dication experimentally $[16,17]$ was unsuccessful.

The purpose of this note is to suggest that it is possible to avoid the variational method and consequent multi-center integrations entirely and that the Schroedinger equation can be solved exactly for a sequence of Hamiltonians that converge to the correct one. This note is intended as a proof of principle, and deals only with the first Hamiltonian in the sequence; it is equivalent to keeping only the hydrogen $1 \mathrm{~s}$ state and is simple enough that most of the calculations can be done "by hand". We also assume that the ion forms an isosceles triangle

and the ground-state energy (ignoring hyperfine effects) is examined as a function of a side and adjacent angle. Even so, the results lie reasonably close to the most recent values.

\section{Hydrogen atom}

Let the one-electron Hamiltonian of an atom ( or indeed, any system) be

$$
H=p^{2}+V(\vec{r})
$$

and have bound-state eigenfunctions and energy levels $\left\{\phi_{a}, E_{a}\right\}$. Then, by completeness,

$$
V(\vec{r})=\sum_{a} v_{a}(\vec{r})<\phi_{a}\left|, \quad v_{a}(\vec{r})=V(\vec{r})\right| \phi_{a}>.
$$

Thus, if we ignore the continuum states, which will be of no interest in the sequel, then we have a sequence of Hamiltonians

$$
H_{n}=p^{2}+\sum_{m=0}^{n} v_{m}<\phi_{m} \mid
$$

and it is easily checked that the Schroedinger equation (we adopt units: $\hbar=$ $2 m=e^{2} / 2=1$ )

$$
\begin{gathered}
\left\{-\nabla^{2}-E\right\} \psi(\vec{r})=-\sum_{m=0}^{n} v_{m}(\vec{r}) \lambda_{m} \\
\lambda_{m}=\int d \vec{s} \phi_{m}^{*}(\vec{s}) \psi(\vec{s})
\end{gathered}
$$

has precisely the first $n$ of the eigenstates of $H$. By transforming to momentum space and writing $E=-\epsilon<0$ (since we are only concerned with bound states) (4) becomes the integral equation

$$
\hat{\psi}(\vec{k})=\sum_{m=0}^{n} \frac{\hat{v}_{m}(\vec{k}) \lambda_{m}}{\epsilon+k^{2}} .
$$


Next, by the Parseval relation for the Fourier transform [2] we have the consistency condition

$$
\begin{gathered}
\lambda_{q}=\frac{1}{(2 \pi)^{3}} \int \hat{\phi}_{q}^{*}(\vec{k}) \hat{\psi}(\vec{k}) d \vec{k}=\sum_{m} A_{q m} \lambda_{m} \\
A_{q m}=\frac{1}{(2 \pi)^{3}} \int \frac{\hat{v}_{m}(\vec{k}) \hat{\phi}_{q}^{*}(\vec{k})}{k^{2}+\epsilon} d \vec{k} .
\end{gathered}
$$

That is to say, the energy levels $\epsilon$ and corresponding $\lambda$ 's are determined by the matrix equations

$$
(\mathbf{A}-\mathbf{I}) \Lambda=0, \quad \operatorname{Det}|\mathbf{A}-\mathbf{I}|=0
$$

where $\Lambda$ is the column vector $\left(\lambda_{0}, \cdots, \lambda_{n}\right)^{T}$. In the next section we illustrate this by working out $H_{0}$ and $H_{1}$ for hydrogen.

It should be pointed out that in (1.2) the $\phi_{a}$ may be any complete set of functions, not just the eigenfunctions of $H$, though the low order approximations are unlikely to be as accurate.

\section{$2.1 \quad$ Hydrogen atom}

The lowest two bound-state wave functions for Hydrogen are[3]

$$
\phi_{0}(r)=\pi^{-1 / 2} e^{-r}, \quad \epsilon_{0}=1, \text { and } \phi_{1}(r)=(32 \pi)^{-1 / 2}(2-r) e^{-r / 2}, \quad \epsilon_{1}=\frac{1}{4} .
$$

Hence,

$$
\begin{array}{cc}
\hat{\phi}_{0}(k)=\frac{8 \sqrt{\pi}}{\left(k^{2}+1\right)^{2}}, & \hat{\phi}_{1}(k)=\frac{32 \sqrt{2 \pi}\left(4 k^{2}-1\right)}{\left(4 k^{2}+1\right)^{3}} \\
\hat{v}_{0}(k)=\frac{8 \sqrt{\pi}}{\left(k^{2}+1\right)}, & \hat{v}_{1}(k)=\frac{8 \sqrt{2 \pi}\left(4 k^{2}-1\right)}{\left(4 k^{2}+1\right)^{2}}
\end{array}
$$

and the matrix elements of $\mathbf{A}$ are $\left(\epsilon=x^{2}\right)$

$$
\begin{gathered}
A_{00}=2 \frac{3+x}{(1+x)^{3}} \\
A_{11}=2 \frac{8 x^{3}+20 x^{2}+6 x+7}{(2 x+1)^{5}} \\
A_{01}=\frac{32 \sqrt{2}}{27} \frac{2 x^{2}+5 x-7}{(1+x)(1+2 x)^{3}} \\
A_{10}=\frac{8 \sqrt{2}}{27} \frac{4 x^{2}+12 x-7}{(1+x)^{2}(1+2 x)^{2}} .
\end{gathered}
$$

It is not difficult to check that the determinant in (1.7) has the form $(x-1)(2 x-$ 1) $P(x) / Q(x)$ where $P$ and $Q$ are polynomials with positive coefficients, so its sole positive real roots are $x=1 / 2$ and $x=1$. The first equation (1.7) has the solution $\lambda_{0}=1, \lambda_{1}=A_{10} /\left(1-A_{00}\right)$ and it is straightforward to check that inverting (1.5) with these values of $x$ reproduces (2.1). 


\section{Triangular Molecule}

Consider the three-proton system, in the Born-Oppenheimer approximation, where one lies at the origin and two lie in the $x-z$-plane at positions

$$
\vec{R}_{ \pm}=R(\cos \alpha, 0, \pm \sin \alpha) .
$$

The Schroedinger equation for an electron subject to this configuration is

$$
-\left(\nabla^{2}+E\right) \psi(\vec{r})=\left[V(\vec{r})+V\left(\vec{r}-\vec{R}_{+}\right)+V\left(\vec{r}-\vec{R}_{-}\right)\right] \psi(\vec{r}) .
$$

Setting $E=-\epsilon,(3.2)$ has the immediate solution in momentum space

$$
\hat{\psi}(\vec{k})=\frac{\hat{v}_{0}(\vec{k})}{k^{2}+\epsilon}\left[\lambda_{0}+e^{i \vec{k} \cdot \vec{R}_{+}} \lambda_{+}+e^{i \vec{k} \cdot \vec{R}_{-}} \lambda_{-}\right]
$$

with

$$
\begin{gathered}
\lambda_{0}=\frac{1}{(2 \pi)^{3}} \int d \vec{k} \hat{\phi}_{0}(\vec{k})^{*} \hat{\psi}(\vec{k}) \\
\lambda_{ \pm}=\frac{1}{(2 \pi)^{3}} \int d \vec{k} \hat{\phi}_{0}(\vec{k})^{*} e^{-i \vec{k} \cdot \vec{R}_{ \pm}} \hat{\psi}(\vec{k}) .
\end{gathered}
$$

Therefore, by defining the four integrals

$$
\begin{gathered}
I_{0}=\frac{1}{(2 \pi)^{3}} \int d \vec{k} \frac{\hat{\phi}_{0}^{*}(\vec{k}) \hat{v}_{0}(\vec{k})}{k^{2}+\epsilon} \\
I_{ \pm}=\frac{1}{(2 \pi)^{3}} \int d \vec{k} \frac{\hat{\phi}_{0}^{*}(\vec{k}) \hat{v}_{0}(\vec{k})}{k^{2}+\epsilon} e^{i \vec{k} \cdot \vec{R}_{ \pm}} \\
I_{1}=\frac{1}{(2 \pi)^{3}} \int d \vec{k} \frac{\hat{\phi}_{0}^{*}(\vec{k}) \hat{v}_{0}(\vec{k})}{k^{2}+\epsilon} e^{-i \vec{k} \cdot\left(\vec{R}_{+}-\vec{R}_{-}\right)},
\end{gathered}
$$

we have the three consistency equations

$$
\begin{aligned}
& \lambda_{0}=I_{0} \lambda_{0}+I_{+} \lambda_{+}+I_{-} \lambda_{-} \\
& \lambda_{+}=I_{+}^{*} \lambda_{0}+I_{0} \lambda_{+}+I_{1} \lambda_{-} \\
& \lambda_{-}=I_{-} \lambda_{0}+I_{1}^{*} \lambda_{+}+I_{0} \lambda_{-} .
\end{aligned}
$$

The four integrals (3.5) are real, $I_{+}=I_{-}$and is independent of $\alpha$. From (3.6) we see that the ground state energy $\epsilon$ is fixed by the determinantal equation $\left(\epsilon=x^{2}\right)$

$$
F[R, \epsilon] \equiv\left(I_{0}-I_{1}-1\right)\left[\left(I_{0}-1\right)^{2}+\left(I_{0}-1\right) I_{1}-2 I_{+}^{2}\right]=0 .
$$

The integrals (3.5) are elementary:

$$
I_{0}=\frac{2(3+x)}{(1+x)^{3}}
$$




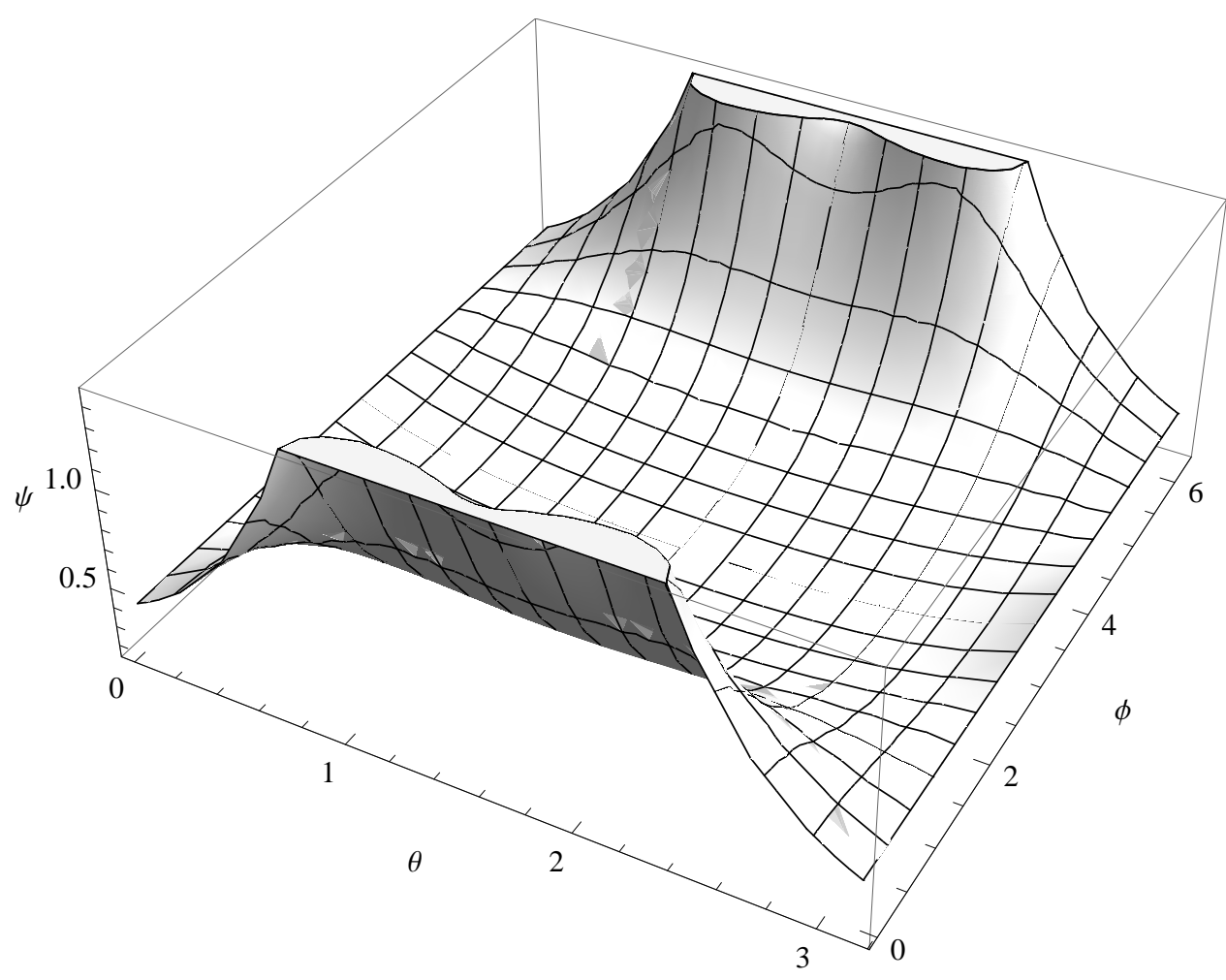

Figure 1: $\psi(1.6, \theta, \phi)$ for $0<\theta<\pi, 0<\phi<2 \pi$.

$$
\begin{gathered}
I_{ \pm}=f(R, x)=\frac{16}{\left(x^{2}-1\right)^{2}}\left\{\frac{e^{-R}-e^{-R x}}{R\left(x^{2}-1\right)}+\frac{1}{8} e^{-R}\left[x^{2}-5+R\left(x^{2}-1\right)\right]\right\} \\
I_{1}=f(2 R \sin \alpha, x) .
\end{gathered}
$$

The spatial wave function is obtained through the Fourier inversion of (3.3) by which we find in spherical coordinates

$$
\begin{gathered}
\psi(r, \theta, \phi)= \\
N\left\{\frac{e^{-r}-e^{-x r}}{r}+\left(\frac{1-I_{0}}{2 I_{+}}\right)\left[\frac{e^{-\rho_{+}}-e^{-x \rho_{+}}}{\rho_{+}}+\frac{e^{-\rho_{-}}-e^{-x \rho_{-}}}{\rho_{-}}\right]\right\},
\end{gathered}
$$

where $N$ is a normalization factor and

$$
\rho_{ \pm}=\sqrt{r^{2}+r^{2}-2 r R(\sin \theta \cos \varphi \cos \alpha \pm \cos \theta \sin \alpha)} .
$$

The wave function in the equilateral triangle configuration for $r=R=1.6$, is shown as a function of $\theta$ and $\varphi$ in Fig.1. 


\section{Results and discussion}

The ground state energy $E=-\epsilon=-x^{2}$ is given by the largest positive root $x$ of (3.7) which is that of the second factor. This is most easily determined graphically and the results for four cases are given below.

\subsection{Linear configuration: $\alpha=\pi / 2$.}

$\begin{array}{cccc}R & x & R & x \\ 0 & 2.1349367 & 1.2 & 1.8036179 \\ 0.1 & 2.1307780 & 1.4 & 1.7347596 \\ 0.2 & 2.1187612 & 1.6 & 1.6694538 \\ 0.3 & 2.0999631 & 1.8 & 1.6082098 \\ 0.4 & 2.0756736 & 2.0 & 1.5512046 \\ 0.5 & 2.0471617 & 2.2 & 1.4984244 \\ 0.6 & 2.0155626 & 2.4 & 1.4497481 \\ 0.7 & 1.9818363 & 2.6 & 1.4049973 \\ 0.8 & 1.9467667 & 2.8 & 1.3639650 \\ 1.0 & 1.8749557 & 3.0 & 1.3264326\end{array}$

The ground-state energy vs $R$ for $\alpha=\pi / 2$ is shown in Fig.2 and the total molecular energy in Fig.3; the ion is unstable.

\subsection{Equilateral triangle $\alpha=\pi / 6$}

$\begin{array}{cccc}R & x & R & x \\ 0.0 & 2.1349367 & 1.2 & 1.9095172 \\ 0.1 & 2.1328424 & 1.4 & 1.8497556 \\ 0.2 & 2.1266444 & 1.6 & 1.7893258 \\ 0.3 & 2.1165658 & 1.8 & 1.7294701 \\ 0.4 & 2.1029191 & 2.0 & 1.6711139 \\ 0.5 & 2.0860668 & 2.2 & 1.6149246 \\ 0.6 & 2.0663935 & 2.4 & 1.5613632 \\ 0.7 & 2.0442855 & 2.6 & 1.5107287 \\ 0.8 & 2.0201173 & 2.8 & 1.4631940 \\ 1.0 & 1.9669892 & 3.0 & 1.4188350\end{array}$

The ground-state and total energies are shown as functions of $R$ in Fig.2 and Fig. 3 For this geometry with $R=1.68$ the exact ground-state energy $x=$ 1.95426 has been proposed[15]. Our value at this spacing is $x=1.76526 \mathrm{a}$ difference of just under 10\%. In Fig.4. we show our result for the total energy Et compared to a recent study of the equilateral case by Medel-Cobaxin et al.[18]

\subsection{Isoceles cases: $\alpha=\pi / 3, \pi / 8$}

For completeness we show the total molecular energy for an obtuse $(\alpha=\pi / 3)$ and an acute $(\alpha=\pi / 8)$ triangular configuration in Figs.2-3. Again, in neither 


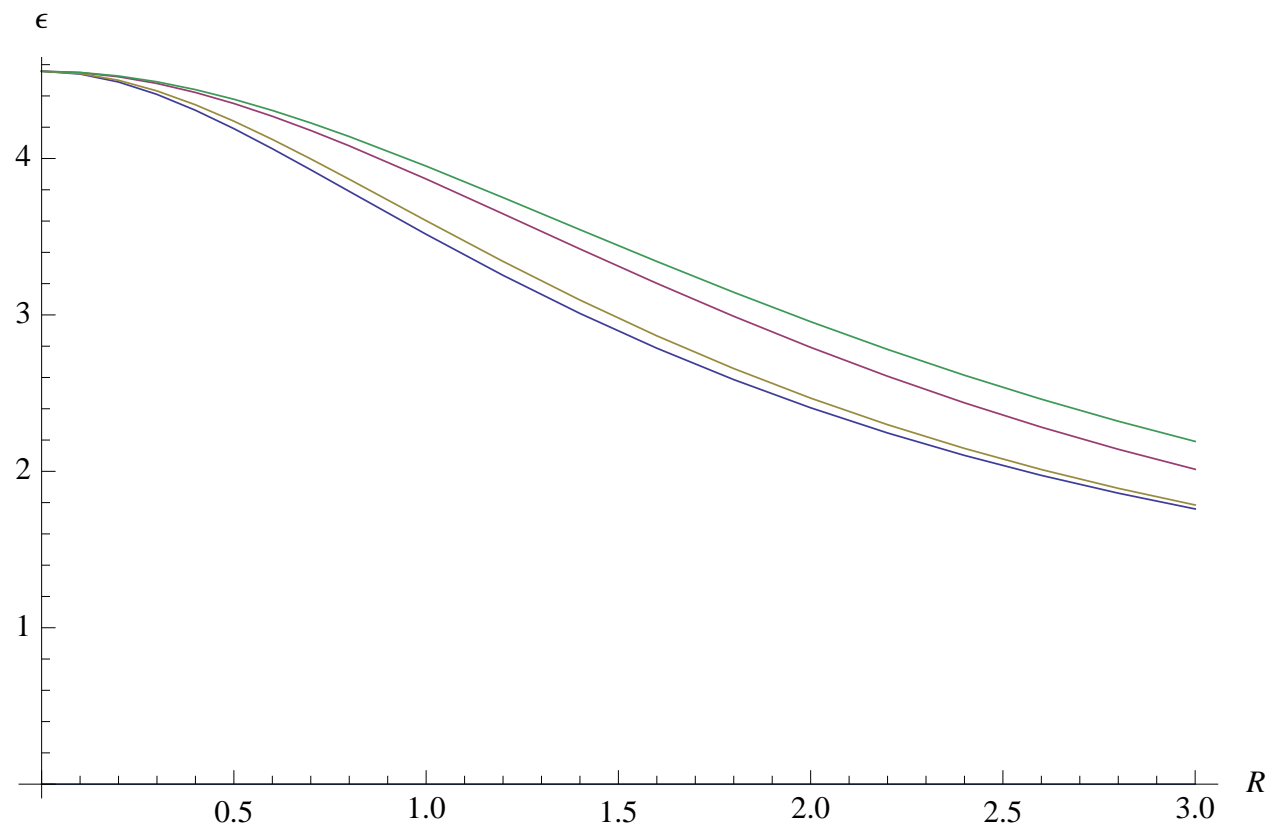

Figure 2: Ground-State energy $\epsilon$ vs $R$. From top to bottom at $R=1: \alpha=\pi / 8$, $\alpha=\pi / 6, \alpha=\pi / 3, \alpha=\pi / 2$ 


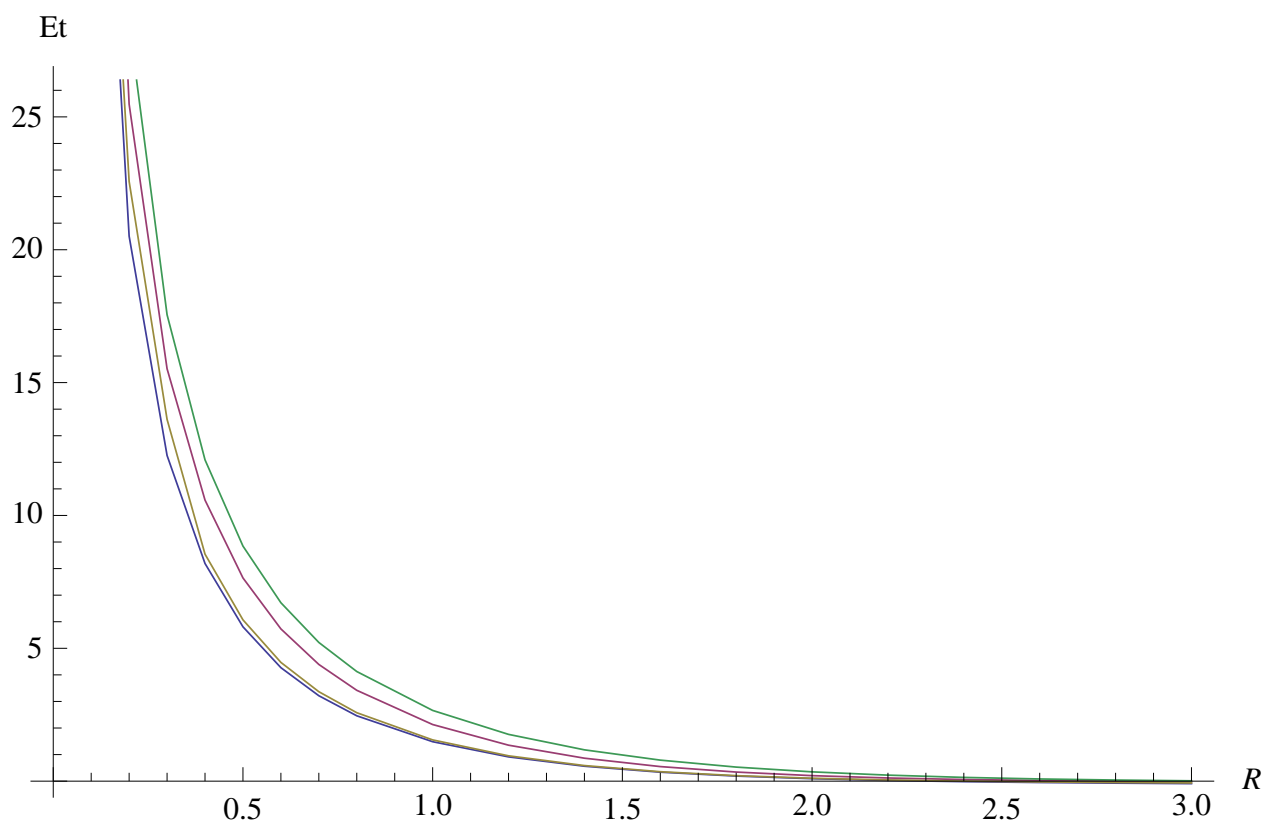

Figure 3: Total energy vs. $R$ : Same order as in Fig.1.

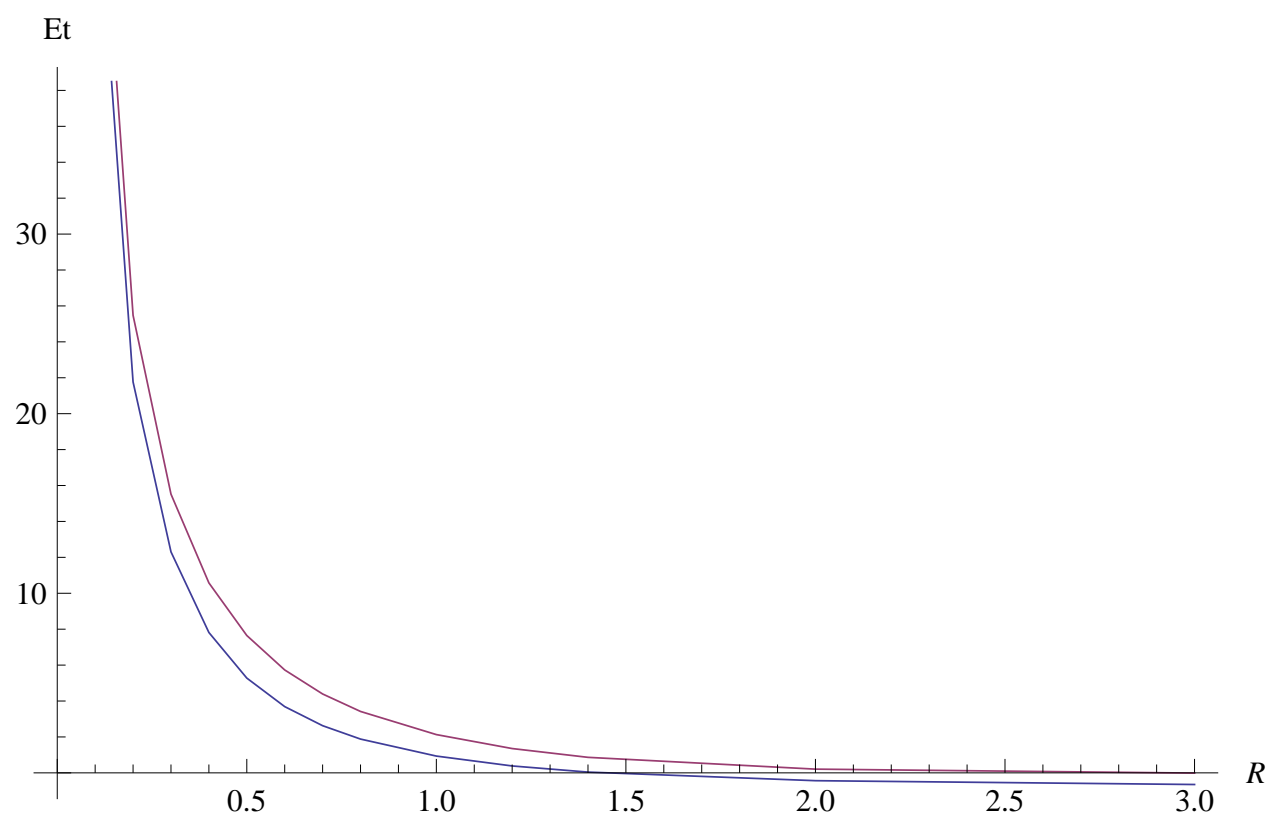

Figure 4: Total energy vs $R$ :Comparison with Ref.[18]. Upper curve-this work. 
case is the ion stable.

$\alpha=\pi / 3$

$\begin{array}{cccc}R & x & R & x \\ 0.0 & 2.1349367 & 1.2 & 1.8281244 \\ 0.1 & 2.1314613 & 1.4 & 1.7592914 \\ 0.2 & 2.1213259 & 1.6 & 1.6929713 \\ 0.3 & 2.1052384 & 1.8 & 1.6300407 \\ 0.4 & 2.0840921 & 2.0 & 1.5709739 \\ 0.5 & 2.0588167 & 2.2 & 1.5159789 \\ 0.6 & 2.0302939 & 2.4 & 1.4650897 \\ 0.7 & 1.9993156 & 2.6 & 1.4182289 \\ 0.8 & 1.9665685 & 2.8 & 1.3752500 \\ 1.0 & 1.8979968 & 3.0 & 1.3359649\end{array}$

$\alpha=\pi / 8$

$\begin{array}{cccc}R & x & R & x \\ 0.0 & 2.1349367 & 1.2 & 1.9365603 \\ 0.1 & 2.1331309 & 1.4 & 1.8828383 \\ 0.2 & 2.1277809 & 1.6 & 1.8280552 \\ 0.3 & 2.1190636 & 1.8 & 1.7733225 \\ 0.4 & 2.1072292 & 2.0 & 1.7194870 \\ 0.5 & 2.0925704 & 2.2 & 1.6671775 \\ 0.6 & 2.0754001 & 2.4 & 1.6168452 \\ 0.7 & 2.0560355 & 2.6 & 1.5688001 \\ 0.8 & 2.0347866 & 2.8 & 1.5232405 \\ 1.0 & 1.9877994 & 3.0 & 1.4802771\end{array}$

In conclusion, we have given the exact solution of the Schroedinger equation, within the Born-Oppenheimer approximation, for a model three-center molecule closely resembling $\mathrm{H}_{3}^{++}$. For the equilateral configuration, where an exact ground-state energy at $R=1.68$ has been proposed[15] the value calculated here agrees to better than $10 \%$.. Furthermore, for our model:

- No multi-center molecular integrals are required.

- The approximation can be systematically improved.

- The corresponding Dirac equation can be solved exactly [20].

- Electric and magnetic fields can be included requiring only the solution of a first or second order ODE.[21]

- It may be feasible to treat the Kohn-Sham equations on the same basis, in which case correlation effects can be included. 


\section{Acknowledgements}

I thank the Universidad de Valladolid, Valladolid, Spain and the DHIP, San Sebastian, Spain, where this work was carried out, for their hospitality. I am also grateful to Prof. Carlos Balbas for comments and references.

\section{References}

[1] J.J. Thomson, Proc. Roy. Soc(Lond)A89, 1 (1913).

[2] T.R. Hogness and E.G. Lunn, Phys. Rev.26, 44 (1925).

[3] G.S. Gordadse, Z.f. Physik 96, 542 (1935).

[4] G.S. Gordadse, Z.f. Physik 99,284 (1936).

[5] C.A. Coulson, Proc. Camb. Phil. Soc. 31, 244 (1935).

[6] G.S. Handler and J.R. Arnold, J. Chem. Phys.27, 144 (1957).

[7] H. Conroy, J. Chem. Phys.41, 1327 (1964).

[8] H. Conroy, J. Chem. Phys.41, 1331 (1964).

[9] H. Conroy, j. Chem. Phys.41, 1336 (1964).

[10] H. Conroy, J. Chem. Phys.51, 3979 (1964).

[11] K. Matsubara, Bul. Nara U. Edu.18, 7 (1969).

[12] R.L. Samorjai and C.P. Yue, J. Chem. Phys.53, 1657 (1970).

[13] R.M. Shoucri and B.T. Darling, J. Chem. Phys.56, 1789 (1973).

[14] J. Hernandez and R. Carbo, J. Chem. Phys.62, 2637 (1975).

[15] J. Ackermann, B. Erdmann and R. Raltzsch, J. Chem. Phys. 101,7 648 (1994).

[16] N. Papp and L. Kervin, Phys.Rev. Lett.23, 1743 (1969).

[17] E.H. Berkowitz and H. Stocker, J. Chem. Phys.55, 4606 (1971).

[18] H. Medel-Cobaxin, A. Alijah and A.V. Turbiner, Coll.Czech Chem. Commun.73,1271 (2008).

[19] A.R. Levin, Reformulation of the Muffin-Tin Problem in Electronic Structure Calculations within the FEAST Framework, [ Ph.D. Thesis, University of Massachusetts, Amherst (2012)].

[20] M.L. Glasser, Relativistic ground-state of the hydrogen molecular ion, Unpublished report.

[21] A.V. Turbiner and J.C. Lopez Vieyra, Phys. Rpts.424, 309 (2006). 